\title{
The correlation of perceived stress and professional concerns during COVID-19 pandemic among Iranian dentists: the mediating role of cognitive flexibility
}

\author{
Afrooz Afshari ${ }^{1}$, Seyedehsareh Hashemikamangar ${ }^{1}$, Sedighe Sadat Hashemikamangar ${ }^{2}$ * \\ ${ }^{1}$ Department of psychology, Alzahra University, Tehran, Iran \\ ${ }^{2}$ Department of operative dentistry, Dental school, Tehran University of medical sciences, Tehran, Iran
}

\section{Abstract}

Objective: To evaluate the association of perceived stress and dental work-related concerns regarding to COVID-19 by mediating role of cognitive flexibility among dentists during pandemic outbreak of COVID-19 in Iran.

Methods: This study was a cross-sectional survey conducted by means of a confidential on-line survey on 174 Iranian dentists. COVID-19 related questions were divided into three parts: dentists' perspective about their career future, virus contagion and concerns of getting infected COVID-19 from patients, and paucity of information and facilities to control the spread of the virus. Perceived stress and psychological flexibility were assessed by PSS (Cohen et al., 1983) and CFI (Dennis et al., 2010). To analyze the data path analysis method was used.

Results: Perceived stress significantly predicted psychological flexibility (both alternatives and control subscales) and COVID-19 factors related to dental work. In addition, the control subscale significantly predicted dentists' concerns toward their career future and fear of COVID-19 contagion. Hence, control subscale of cognitive flexibility mediated the relationship of perceived stress and professional concerns of dentists during COVID-19 pandemic.

Conclusion: Given these results, elevated stress during an infectious disease outbreak can affect (have an impact on) perceived stress and professional concerns among dentists. Different aspects of dentists' lives can be affected by those concerns and stress. Interventions to enhance cognitive flexibility should be applied to reduce the psychological burden of the crisis for dentists.

Keywords: COVID-19; dentists; professional concerns; perceived stress; psychological flexibility.

Citation: Afshari A, et al. (2020) The correlation of perceived stress and professional concerns during COVID-19 pandemic among Iranian dentists: the mediating role of cognitive flexibility. Dentistry 3000. 1:a001 doi:10.5195/d3000.2020.119 Received: October 28, 2020

Accepted: November 30, 2020

Published: Dezember 22, 2020

Copyright: @2020 Afshari A, et al. This is an open access article licensed under a Creative Commons Attribution Work 4.0 United States License.

Email: smhk58950@gmail.com

\section{Introduction}

\section{The first cases of Coronavirus} (COVID-19) were reported in late December 2019 in Wuhan, China, and after its rapid spread in many countries around the world, on March 11, 2020, the World Health Organization (WHO) declared it as a pandemic. The disease became a global threat quickly. It is the largest outbreak of atypical pneumonia since SARS's acute respiratory syndrome in 2003 . The common transmission routes of coronavirus include direct and contact transmissions such as droplet inhalation transmission, cough and sneeze, contact with oral, nasal, and eye mucous membranes [1]. This respiratory virus can be transmitted from person to person through coarse or small droplets, and can also be transmitted directly or indirectly through saliva [2].

Past researches on smaller-scale epidemics and recent researches on COVID-19 have reported a large amount of stress and increased psychological problems among professionals who dealt with patients suffering from Corona [36]. In a review study conducted on 
experienced stress by health care workers during COVID-19

pandemic, extensive strain due to stressful experiences, depression and anxiety symptoms were reported [7].

Dentists might be one of the most stress vulnerable population during COVID-19 pandemic because of high risk for transmission of infection and disease due to exposure to blood, saliva, and aerosol / droplet generated during dental treatment procedures [2]. Since the virus spread can happen in the absence of clinical symptoms, [8] psychological stress of dental professionals might be more serious due to the ambiguous nature of the virus and the probability of virus contamination in their work environment.

Many studies mentioned the psychological problems of dental staff in work environment or in relation to patients $[9,10]$. In a review study performed on key determinants of health and wellbeing among dentists working in the UK, it was revealed that workplace characteristics were the most often explored determinants by researchers and reported as being a key issue. The results also showed that the health and wellbeing of dentists are associated with a wide range of factors: personal, professional career, relationships, job specification, workplace and system [11]. In another recent study on elevated distress associated with COVID-19 and psychological factors in Israeli dental staff, it was proven that those who had a background illness, higher subjective overload, and fear of contracting COVID-19 from patient, reported higher levels of psychological distress. Being in a committed relationship and having higher scores for selfefficacy was related to lower psychological distress [12].

There are many individual differences between people when they are exposed to threats. The way individuals perceive and interpret a stressful event is considered of greater importance than the event itself. According to Lazarus, perceived stress refers to the degree to which people view life events as unpredictable, uncontrollable, and stressful [13]. Lazarus believes that stimuli that are evaluated as stressor call for stress responses.

However, in a crisis such as the outbreak of an infectious lifethreatening virus that endangers the sense of safety and wellbeing of people, one of the crucial things which can possibly affect the mental health of individuals is cognitive flexibility. The term is described as "the ability to switch cognitive sets to adapt to the changing environmental stimuli" [14]. If a stressful situation is perceived as "beyond a person's control", cognitive flexibility can be reduced by the person's appraisal. Whereas, a brief social stressor can have an enhancing effect on mental flexibility [15]. Therefore, one of the possible reasons for individual differences in cognitive flexibility [16] is the variety of perceptions and appraisal of stressful events.

In this study, we aimed to evaluate the perceived stress and cognitive flexibility of dental staff about COVID-19 factors related to work environment such as concerns about their career future, contagion control and paucity of information, and facilities to control the disease.

\section{Material and Methods}

Design and sample

This study is based on a path analysis designed to test a hypothetical model involving perceived stress, cognitive flexibility, and COVID-19 factors related to work environment among Iranian dentists during Coronavirus outbreak. Using convenient sampling method and 
online questionnaires sent to dentists, data were recruited. Participants were general dentists or specialist in Iran who work in either private office, clinics, or both, and aged between 25 to 65 years old from both genders.

Exclusion criteria were unemployment for at least one year prior to the start of the Covid-19 pandemic and/or unwillingness to participate in the research.

\section{Ethical considerations}

This is a cross-sectional study, which is approved by research ethics Committee of School of Dentistry in Tehran University of Medical Science, Iran (approval code: IR.TUMS.DENTISTRY. REC.1399.025). Before completing the questionnaires, participants signed informed consent forms which contained information about the researchers, the purpose of research, advantages of participation, issues related to confidentiality, and procedures for withdrawal from the research.

\section{Measures}

In this study, to assess perceived stress among dentists, Perceived Stress Scale (PSS) was administered to participants. The PSS, designed by Cohen, Kamarck, \& Mermelstein (1983), has different forms with 10, 4, and 14 questions. In this study, 14questions form was used. Each item is rated on a 5 -point scale $(0=$ Never to 4 =Always). Items are designed to measure the extent to which one's life is perceived as "unpredictable, uncontrollable, and overloading" [17]. The higher a person's score on the test, the higher his/her stress level. Cohen et al. (1983) Reported test-retest reliability of the scale is 0.85 and 0.84 to 0.86 is its internal consistency. Evaluating the psychometric properties of the perceived stress scale in an Iranian sample showed good reliability and validity for PSS [18]. In the present study, the reliability of this scale by means of Cronbach alpha test was 0.91

To measure cognitive flexibility in dentists, the Cognitive Flexibility inventory (Dennis \& Vander Wal, 2010) was used (20 questions). This is a self-report inventory with 7-point Likert (from strongly disagree to strongly agree) inventory with two subscales called the Alternatives and Control subscale. This measurement evaluates the cognitive flexibility that presents the ability to challenge irrational thoughts and replace them with efficient and balanced ones. It measures three aspects of cognitive flexibility: 1 )
The tendency to perceive difficult situations as controllable, 2) The ability to perceive multiple alternative explanations for life occurrences and human behavior, and 3) The ability to generate multiple alternative solutions in difficult situations. The CFI showed high internal consistency (for the total score $\alpha=0.91$, and for Alternatives and Control subscale respectively 0.91 and 0.86 was reported). It showed adequate test-retest reliability over 7-weeks (CFI total score: $r=0.81$;

Alternatives subscale: $r=0.75$; Control subscale: $r=0.77$ ), and good convergent validity with other self-report measures of cognitive flexibility and negative correlations with measures of depression (14). The Internal consistency of CFI in the present study was 0.90 .

COVID-19 factors related to dental environment:

Fear of getting infected COVID-19 from patients/dental environment and virus contagion was measured by two questions: "Are you afraid to be infected with COVID-19 because of your profession?" and "I am worried that because of aerosol/droplets production in the dental environment, it causes contagion and spread of the disease". Responses were coded 
from 0 for "Not at all" to 4 for "Very afraid".

Receiving enough professional knowledge regarding COVID-19 and ability to adhere to valid guidelines were measured by two following questions: "To maintain a safe working environment since the COVID-19 outbreak, do you acquire sufficient knowledge (e.g. lectures, seminars, information leaflet, etc.)?" The second question was "I do not have enough facility to control infection by regarding guidelines and valid instructions". Responses were coded from 0 for "Not at all" to 4 for "Very much".

The last part was concerns of dentists about career future, which was measured by two statements; "Considering the COVID-19 outbreak and uncertain future of this disease, I don't have a clear job prospect", and "Due to the COVID-19 outbreak, even for emergency treatment, I am not reluctant to reopen the office and restart my clinical work". These responses were coded from 0 for "Not at all" to 4 for "Very much".

\section{Demographic questions}

Some self-report questions such as age, gender, marital status, years of work experience, work place( private office/clinic/both), professional status (general dentist, specialist, resident (specialty resident)).

Another question was about being in a risk group: "Are you defined as being in a high-risk population (suffering from asthma/chronic kidney disease and who are undergoing dialysis/liver disease/serious heart conditions/immunocompromised conditions, such as cancer treatment/diabetes)?" The responses were coded as 0 for "No" and 1 for "Yes".

\section{Statistical analysis}

This study used SPSS-20 and AMOS-20 to analyze data. The SPSS software was used to analyze participants' demographic characteristics, correlation between variables, regression analysis and reliability of instruments. AMOS software was used to examine the feasibility of the proposed model, by considering goodness of fit indices such as adjusted goodness of fit index (AGFI), the goodness of fit index (GFI), the comparative-fit index (CFI), the root mean square error of approximation (RMSEA), and the normed fit index (NFI). The fit of the model was examined using $\chi 2 /$ degrees of freedom (d.f.) analyses which should be less than 3.00 , other model fit analysis scores, such as AGFI, GFI, CFI, and $\mathrm{NFI}$, should be greater than 0.80 . The acceptable model fit should be greater than 0.90, and RMSEA should be less than 1.00 .

\section{Results}

\section{Participant characteristics}

The age range of the sample was 24-65 years $(n=174)$ and the average was 38.11 (SD=9.67). $71.3 \%$ of them were female and $28.7 \%$ were male. $35.6 \%$ of the total sample were single/unmarried and $64.4 \%$ were married. $47.7 \%$ of the total sample were general dentists, $40.2 \%$ were professional dentists, and $12.1 \%$ were resident (specialty student). Majority of participants $(n=83$, $47.7 \%$ ) had more than 10 years of work experience, $17.8 \%$ less than 2 years, $14.9 \%$ of respondents $2-5$ years, $19.5 \%$ had $6-10$ years of work experience. More than half of the respondents did not have any child (52.3\%), $25.9 \%$ had one child, $20.1 \%$ had 2 children and $1.7 \%$ had more than 2 children. $61 \%$ of whom had children were women and $39 \%$ men.

Additionally, the majority of the dentists were healthy $(n=157$, $90.2 \%$ ) and $9.8 \%$ were in the highrisk group with a kind of chronic or immunocompromised condition.

\section{Descriptive statistics}


The mean score for the variables of perceived stress was 28.08, for cognitive flexibility was 105.27 , for paucity of information and facilities 3.77, for fear of COVID-19 contagion in work environment 5.83 , and finally for concerns of their career future 4.38 . The standardized skewness and kurtosis did not exceed the critical value $( \pm 1.96)$ at the significance level of 0.05 [19]. The Multivariate critical ratio of the whole sample was 0.688 , which is less than 2.58 . Therefore, the conditions of normal distribution were satisfied.

\section{Correlation among variables}

In this research, there was a significant negative correlation between perceived stress and psychological flexibility (Alternatives subscale, $r=-0.34$, and Control subscale, $r=-0.59$, $\mathrm{p} \leq 0.0001$ ) and significant positive correlation between perceived stress and COVID-19 factors related to the dental environment (fear of COVID-19 contagion $r=$ 0.21 , career future $r=0.24$, $\mathrm{p}<0.01$ ). Additionally, there was a significant negative correlation between control subscale and fear of COVID-19 contagion $r=-0.16$, $p<0.05$; and career future $r=-0.28$, $p \leq 0.0001)$. In general, perceived stress, fear of virus contagion in dental work environment, and control subscale of cognitive flexibility explain $31.2 \%$ of the variance of professional concerns of dentists during the recent crisis.

The results of comparing female and male in the variables of cognitive flexibility, concerns about virus contagion in the dental environment and getting infected COVID-19 from patients, concerns about career future, facilities and dental information from reputable guidelines in preventing disease transmission showed that there was no significant difference between the two genders in any of these variables.

The results of comparing the mentioned variables in dentists with less than 2 years, 2-5 years, 6-10 and more than 10 years of work experience showed that in general there is no significant difference between the groups with different work experience in terms of the mentioned variables. However, the intergroup comparison of perceived stress showed that perceived stress of dentists with less than two years of work experience (mean = 30.194 and standard deviation = 1,574 ) was significantly higher than that of dentists with more than 10 years of work experience (mean $=25.853$ and standard deviation $=1.503$ ) $p=048$. There was no significant difference between perceived stresses in other groups. Moreover, no significant difference was observed between any of the groups of assistants, general dentists and specialists in the research variables.

In addition, in the variable concerns about virus contagion in the dental environment and getting infected COVID-19 from patients, difference between two groups of dentists with more than 10 years of work experience ( $m e a n=5,530$ and standard deviation $=0.198$ ) and dentists with less than two years of work experience $($ mean $=6.378$ and standard deviation $=0.324$ ) was significantly high $\mathrm{p}=.025$.

Furthermore, the concern in group with less than two years of work experience was significantly higher than that of the group with more than 10 years of work experience. There was no significant difference between the other groups. Also, there was no significant difference between the groups in terms of concerns about career future.

Additionally, no significant difference was observed between the dentists working in a private office with those working in a clinic in terms of research variables. 
Dentistry 3000

Vol 8 No 1 (2020) DOI 10.5195/d3000.2020.119

There was no significant difference between dentists with children and dentists without children in the mentioned variables, except for the variable concerns about virus contagion in the dental environment and getting infected COVID-19 from patients. This variable was significantly higher in dentists with more children $(p=.018)$.

No significant relationship was observed between the age of the dentists and the mentioned variables.

\section{Mediation test of cognitive} flexibility

As the Alternative component of Cognitive flexibility did not have any correlation with COVID-19 related variables in dentists, it was removed from the equation.

The model included perceived stress as exogenous variable and control subscale of cognitive flexibility, fear of COVID-19 contagion in the work environment, and career future concerns due to Corona virus outbreak were considered as endogenous variables.

A mediation effect is created when a third variable/construct intervenes between two other related constructs [19]. Tests carried out by path analysis using

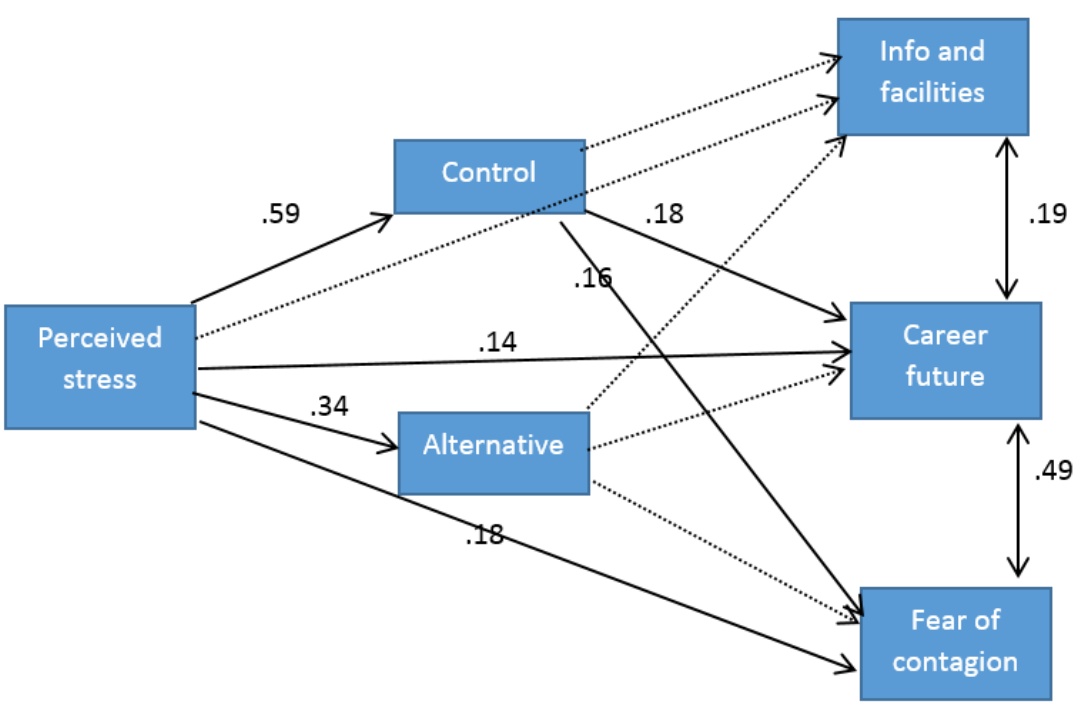

Figure 1. Path diagram

regression methods (SPSS) and structural model (AMOS). The results can describe the final path substructure model, as shown in figure 1.

By considering the Control component of cognitive flexibility of dentists as the mediator variable between perceived stress and career future concerns, the results revealed that although the comparison between the full mediation model and indirect models' goodness-of-fit indices showed that both models fit the data, the indirect model showed a significantly better fit than the full mediation model. In addition, since the indirect model showed smaller AIC (Akaike Information Correction: 18.185) than the full mediation model (Akaike Information Correction: 20.000), and the PNFI (Parsimony Normed Fit Index) of the full mediation model was (0.0) lesser than that of the indirect model (0.166), control component was mediated between perceived stress and career future concerns in dentists. Indirect model goodness of fit indices were (Chi-square: 0.185, p> 0.05, NFI: .99, GFI: 0.99, AGFI: 0.99, CFI: 1.00 , and RMSEA: 0.00).

These results revealed that control subscale of cognitive flexibility is a full mediator between perceived stress and career future concerns. There were direct effects between perceived stress and fear of COVID-19 contagion in the work environment, as well as perceived stress and career future (Table 1). 
Table 1. Direct, indirect and total effects for the model $(n=174)$

\begin{tabular}{|c|c|c|c|c|c|c|c|c|}
\hline $\begin{array}{l}\text { Predictive } \\
\text { Variable }\end{array}$ & $\begin{array}{c}\text { Dependent } \\
\text { Variable }\end{array}$ & Beta & Sig & CR & SE & $\begin{array}{l}\text { Direct effect } \\
\text { (p value) }\end{array}$ & $\begin{array}{c}\text { Indirect effect ( } p \\
\text { value) }\end{array}$ & $\begin{array}{l}\text { Full mediation } \\
\text { ( } p \text { value) }\end{array}$ \\
\hline $\begin{array}{l}\text { Perceived } \\
\text { stress }\end{array}$ & Control & -.59 & $<.00001$ & -9.51 & .06 & 0 & $\begin{array}{c}-.59 \\
(<.0001)\end{array}$ & $-.59(<.0001)$ \\
\hline $\begin{array}{l}\text { Perceived } \\
\text { stress }\end{array}$ & Career future & .24 & .001 & .43 & .02 & $\begin{array}{l}.14 \\
(.03)\end{array}$ & 0 & $\begin{array}{l}.034 \\
(.67)\end{array}$ \\
\hline control & Career future & -.28 & $<.00001$ & -2.33 & .02 & 0 & $\begin{array}{c}-.20 \\
(.002)\end{array}$ & $\begin{array}{c}.18 \\
(.020)\end{array}$ \\
\hline $\begin{array}{l}\text { Perceived } \\
\text { stress }\end{array}$ & $\begin{array}{l}\text { Contagion } \\
\text { fear }\end{array}$ & .21 & .005 & 2.83 & .019 & $\begin{array}{c}.18 \\
(.02)\end{array}$ & $\begin{array}{c}.21 \\
(.005)\end{array}$ & $\begin{array}{c}.21 \\
(005)\end{array}$ \\
\hline Control & $\begin{array}{l}\text { Contagion } \\
\text { fear }\end{array}$ & -.16 & .03 & -.65 & .020 & 0 & $\begin{array}{l}-.16 \\
(.03)\end{array}$ & $\begin{array}{l}-.06 \\
(.52)\end{array}$ \\
\hline $\begin{array}{l}\text { Contagion } \\
\text { fear }\end{array}$ & Career future & .52 & $<.00001$ & 7.50 & .075 & $\begin{array}{c}.49 \\
(<.00001)\end{array}$ & $\begin{array}{c}.49 \\
(<.00001)\end{array}$ & $\begin{array}{c}.49 \\
(<.00001)\end{array}$ \\
\hline
\end{tabular}

CR: critical ratio; SE: standard error

Moreover, the control component was mediated the relationship between perceived stress and fear of COVID-19 transmission in the dental work environment. Indirect model goodness of fit indices were (Chi-square: $3.65, p>0.05$, NFI: .97, GFI: 0.99, AGFI: 0.90, CFI: .98).

\section{Discussion}

The first result of this study indicated that Iranian dentists are under high levels of stress during the COVID-19 pandemic. They reported concerns about being infected by a new virus while presenting professional services to patients. This result was similar to those previous studies conducted among dentists, nurses and medical staff population $[3,5,6$, 12]. A significant amount of stress and mental health problems among the specialists was shown in those studies.
Due to the unknown nature of the disease, lack of vaccine, and high probability of virus transmission in dental workplaces [2], dentists are prone to experience high levels of stress and be reluctant to provide dental services to patients.

As high levels of stress can reduce the proper function of the individuals, determining the factors that lead to stress reduction is of significant importance. In this study, we investigated the effect of perceived stress of dental staff 
during COVID-19 pandemic on

their professional stress and

concerns. This effect was

mediated by control component

of cognitive flexibility. In fact, when a dentist perceives her/his as being enough compatible to have control over changeable and challenging circumstances, he/she would experience less distress and concerns toward the profession.

Although the control component of cognitive flexibility could predict the professional stress in dentists, it was not a very strong predictive factor. However, fear of COVID-19 contagion in the work environment was a better predictive variable for it. According to previous studies which mentioned the effective and significant role of environmental workplace of dentists $[9,11,12]$ on their mental health, the authors declare that during the COVID-19 pandemic, mental health of Iranian dentists should be given serious attention. Although by means of dentists' reports, there was no significant correlation between lack of information and facilities with their career future concerns about the upcoming circumstances, the other related variables such as contextual factors should be investigated.

Perceived stress among dentists with less than two years of work experience was greater than that of dentists with a longer work experience, and the concerns about virus contagion in the dental environment and getting infected COVID-19 from patients was higher among dentists with less than two years of work experience. This finding is in agreement with Farrukh et al. 20. According to these findings, young people who have less clinical work experience do not seem to have the confidence to provide the condition to control disease transmission. On the other hand, more work experience may have led to false confidence in more experienced dentists. This needs further investigation.

More concerns about virus contagion in the dental environment and getting infected COVID-19 from have been reported in dentists with more children. It makes sense because infections would mean either risking hurting one's children through unintentional infection or being isolated from one's children for two weeks (which can feel longer based on how young ones children are). Given the fact that most of the participants in the present study who had children were women (61\%), and in Iranian society women are more responsible for taking care of their children, job concerns in these people may be doubled that affect the perception of their career future.

The author tried their best to follow up with these survey respondents in two years or so to see if the pandemic has had a lasting effect on how they view/treat infection, or if their professional anxieties were proven or not.

\section{Conclusion}

Given these results, elevated stress during an infectious disease outbreak can affect (have an impact on) perceived stress and career future concerns among dentists. Different aspects of dentists' lives can be affected by those concerns and stress. Interventions to enhance cognitive flexibility should be applied to reduce the psychological burden of the crisis for dentists.

\section{Conflicts of interest}

The authors declare no competing interest.

\section{References}


1. 2019-nCoV transmission through the ocular surface must not be ignored. Lu C-w, Liu X-f, Jia Z-f. Lancet (London, England). 2020;395(10224):e39.

2. Transmission routes of 2019-nCoV and controls in dental practice. Peng $X, X u X, L i Y$, Cheng L, Zhou X, Ren B. International Journal of Oral Science. 2020;12(1):1-6.

3. COVID-19 pandemic and its impact on mental health of healthcare professionals. Tsamakis K, Rizos E, Manolis AJ, Chaidou S, Kympouropoulos S, Spartalis E, et al. [Comment] Experimental and Therapeutic Medicine. 2020;19(6):3451-3.

4. Factors Associated with Resilience Among Medical Staff in Radiology Departments During The Outbreak of 2019 Novel Coronavirus Disease (COVID-19): A Cross-Sectional Study. Huang L, Wang Y, Liu J, Ye PF, Cheng BC, Xu HY, et al. Medical Science Monitor. 2020;26.

5. COVID-19 Pandemic: Stress Experience of Healthcare Workers A Short Current Review. Bohlken J, Schomig F, Lemke MR, Pumberger $M$, Riedel-Heller SG. Psychiatrische Praxis. 2020;47(4):190-7.
6. Psychological status of surgical staff during the COVID-19 outbreak. Xu J, Xu QH, Wang CM, Wang J. Psychiatry Research. 2020;288.

7. Impact of COVID-19 on the mental health of surgeons and coping strategies. Balasubramanian A, Paleri V, Bennett R, Paleri V. Head and Neck-Journal for the Sciences and Specialties of the Head and Neck.

8. Incubation period of 2019 novel coronavirus (2019-nCoV) infections among travellers from Wuhan, China, 20-28 January 2020. Backer JA, Klinkenberg D, Wallinga J. Eurosurveillance. 2020;25(5):2000062.

\section{Assessment of Stress among Doctors of Dental Medicine. Jahić IM, Bukejlović J, Alić-Drina S, Nakaš E. Acta Stomatologica Croatica. 2019;53(4):354.}

10. Self-reported psychological problems amongst undergraduate dental students: A pilot study in seven European countries. Stangvaltaite-Mouhat L, Pūrienè A, Chałas R, Hysi D, Katrova L, Nacaite $M$, et al. European Journal of Dental Education. 2020;24(2):341-50.
11. Key determinants of health and wellbeing of dentists within the UK: a rapid review of over two decades of research. Salazar FBC, Sipiyaruk K, White S, Gallagher JE. British dental journal. 2019;227(2):127-36.

12. COVID-19 Factors and Psychological Factors Associated with Elevated Psychological Distress among Dentists and Dental Hygienists in Israel. Shacham M, Hamama-Raz $Y$, Kolerman R, Mijiritsky O, BenEzra M, Mijiritsky E. International Journal of Environmental Research and Public Health. 2020;17(8):2900.

13. Stress and emotion: A new synthesis. Lazarus RS. Springer Publishing Company; 2006.

14. The cognitive flexibility inventory: Instrument development and estimates of reliability and validity. Dennis JP, Vander Wal JS. Cognitive therapy and research. 2010;34(3):241-53.

15. Acute stressor effects on cognitive flexibility: Mediating role of stressor appraisals and cortisol. Gabrys RL, Howell JW, Cebulski SF, Anismana $\mathrm{H}$, Matheson K. Stress. 2019;22(2):182-9. 
Vol 8 No 1 (2020) DOI 10.5195/d3000.2020.119

16. The role of stress mindset in shaping cognitive, emotional, and physiological responses to challenging and threatening stress. Crum AJ, Akinola $M$, Martin A, Fath S. Anxiety, stress, \& coping. 2017;30(4):379-95.

17. A global measure of perceived stress. Cohen $\mathrm{S}$, Kamarck $T$, Mermelstein R. Journal of health and social behavior. 1983:385-96.

18. Assessing Stress in Cancer Patients: Factorial Validity of the Perceived Stress Scale in Iran. Journal of Nursing Education. 2014;2(1):13-22.

19. Multivariate data analysis . Uppersaddle River. Hair JF, Black WC, Babin BJ, Anderson RE, Tatham R. NJ: Pearson Prentice Hall; 2006. 